\title{
Article
}

\section{Ambient Cumulative PM2.5 Exposure and the Risk of Lung Cancer Incidence and Mortality: A Retrospective Cohort Study}

\author{
Hung-Ling Huang ${ }^{1,2,3,4,5} \mathbb{D}$, Yung-Hsin Chuang ${ }^{6}$, Tzu-Hsuan Lin ${ }^{6}$, Changqing Lin ${ }^{7} \mathbb{D}$, Yen-Hsu Chen ${ }^{3,8,9} \mathbb{D}$, \\ Jen-Yu Hung ${ }^{1,2,3,5}$ and Ta-Chien Chan ${ }^{6,10, *(D)}$
}

1 Kaohsiung Municipal Ta-Tung Hospital, Kaohsiung 801, Taiwan; 990325kmuh@gmail.com (H.-L.H.); jenyuhung@gmail.com (J.-Y.H.)

2 Division of Pulmonary and Critical Care Medicine, Kaohsiung Medical University Hospital, Kaohsiung Medical University, Kaohsiung 807, Taiwan

3 Department of Internal Medicine, Kaohsiung Medical University Hospital, Kaohsiung Medical University, Kaohsiung 807, Taiwan; infchen@gmail.com

4 Graduate Institute of Medicine, College of Medicine, Kaohsiung Medical University, Kaohsiung 807, Taiwan

5 School of Medicine, College of Medicine, Kaohsiung Medical University, Kaohsiung 807, Taiwan

6 Research Center for Humanities and Social Sciences, Academia Sinica, Taipei 115, Taiwan;

yhchuang.claire@gmail.com (Y.-H.C.); sharonlin0822@gmail.com (T.-H.L.)

7 Division of Environment and Sustainability, The Hong Kong University of Science and Technology, Hong Kong, China; cqlin@ust.hk

8 School of Medicine, Graduate Institute of Medicine, Sepsis Research Center, Center of Tropical Medicine and Infectious Diseases, Kaohsiung Medical University, Kaohsiung 807, Taiwan

9 Department of Biological Science and Technology, College of Biological Science and Technology, National Yang Ming Chiao Tung University, Hsinchu 300, Taiwan

check for

updates

Citation: Huang, H.-L.; Chuang, Y.-H.; Lin, T.-H.; Lin, C.; Chen, Y.-H.; Hung, J.-Y.; Chan, T.-C. Ambient Cumulative PM2.5 Exposure and the Risk of Lung Cancer Incidence and Mortality: A Retrospective Cohort Study. Int. J. Environ. Res. Public Health 2021, 18, 12400. https:// doi.org/10.3390/ijerph182312400

Academic Editor: John Cherrie

Received: 18 October 2021

Accepted: 22 November 2021

Published: 25 November 2021

Publisher's Note: MDPI stays neutral with regard to jurisdictional claims in published maps and institutional affiliations.

Copyright: (C) 2021 by the authors. Licensee MDPI, Basel, Switzerland. This article is an open access article distributed under the terms and conditions of the Creative Commons Attribution (CC BY) license (https:/ / creativecommons.org/licenses/by/ $4.0 /)$.
10 Institute of Public Health, School of Medicine, National Yang Ming Chiao Tung University, Taipei 112, Taiwan

* Correspondence: tachien@gate.sinica.edu.tw; Tel.: +886-2-27-898-160

\begin{abstract}
Smoking, sex, air pollution, lifestyle, and diet may act independently or in concert with each other to contribute to the different outcomes of lung cancer (LC). This study aims to explore their associations with the carcinogenesis of LC, which will be useful for formulating further preventive strategies. This retrospective, longitudinal follow-up cohort study was carried out by connecting to the MJ Health Database, Taiwan Cancer Registry database, and Taiwan cause of death database from 2000 to 2015. The studied subjects were persons attending the health check-ups, distributed throughout the main island of Taiwan. Cox proportional hazards regression models were used to investigate the risk factors associated with LC development and mortality after stratifying by smoking status, with a special emphasis on ambient two-year average $\mathrm{PM}_{2.5}$ exposure, using a satellite-based spatiotemporal model at a resolution of $1 \mathrm{~km}^{2}$, and on dietary habit including consumption of fruits and vegetables. After a median follow-up of 12.3 years, 736 people developed LC, and 401 people died of LC-related causes. For never smokers, the risk of developing LC (aHR: 1.32, 95\%CI: 1.12-1.56) and dying from LC-related causes (aHR: 1.28, 95\%CI: 1.01-1.63) rises significantly with every $10 \mu \mathrm{g} / \mathrm{m}^{3}$ increment of $\mathrm{PM}_{2.5}$ exposure, but not for ever smokers. Daily consumption of more than two servings of vegetables and fruits is associated with lowering LC risk in ever smokers (aHR: 0.68, 95\%CI: 0.47-0.97), and preventing $\mathrm{PM}_{2.5}$ exposure is associated with lowering LC risk for never smokers.
\end{abstract}

Keywords: fine particulate matter; lung cancer; health behaviors; diet

\section{Introduction}

Lung cancer (LC) is the most common cancer in the world, with 2.2 million new cases in 2019, and it is by far the leading cause of cancer death among both men and women, making up $25 \%$ of all cancer deaths [1]. The overall incidence of LC in Taiwan ranks 15th globally and is the second highest in Asia, trailing only North Korea [1]. It has consistently 
been the leading cause of cancer-related death in Taiwan since 2011, with a mortality rate of 41.1 per 100,000 people in 2019 [2].

A synergic interaction of myriad risk factors contributes to the development of LC, including environmental toxin exposure, genetic predisposition, infectious comorbidities, and individual lifestyle. Despite a decline in incidence and mortality of LC following global tobacco control policies [3,4], LC among never smokers still accounts for an estimated $20 \%$ of cases in men and more than $50 \%$ of cases women [4,5]. Additionally, the global incidence of lung adenocarcinoma is still increasing against the trend of smoking rates in both sexes [4,6-9]. We can, therefore, speculate that tobacco smoke accounts for only a minority of LC development among nonsmokers. The corresponding effect of exposure to ambient fine particulate matter $\left(\mathrm{PM}_{2.5}\right)$ on the development of LC and LC-related mortality, particularly in patients who have never smoked, has been demonstrated in several studies [10-14]. It is known that the impact of $\mathrm{PM}_{2.5}$ on LC development is greater in Asia than in Western countries [15]. In Taiwan, never smokers constitute a major proportion of LC patients, up to $50 \%$ and $90 \%$ in males and females, respectively $[2,10]$.

A nationwide study, using a registry database, conducted in Taiwan has demonstrated a steadily increasing age-adjusted incidence of lung adenocarcinoma in male never smokers, from 9.06 to 23.25 per 100,000 population as the smoking rate decreased from 59.4 to $29.9 \%$, and from 7.05 to 24.22 per 100,000 population in never smoker females from 1995 to 2015. The accelerated increase in $\mathrm{PM}_{2.5}$ levels, particularly in southern Taiwan, might be a possible explanation [10]. However, that retrospective study did not consider the impact of personal lifestyle and couldn't reflect the temporal relationship between $\mathrm{PM}_{2.5}$ and LC development.

The impact of dietary factors on LC development is uncertain. A negative association between the consumption of $\beta$-carotene-, vitamin C-rich vegetables and fruits and LC development has been reported in never smoker females [16-18], while the opposite trend of LC increment was noted among smokers with dietary supplementation of betacarotene [19].

With the gradual implementation of LC prevention policies around the world, this retrospective, longitudinal follow-up study has two aims: (1) to evaluate and compare the risk factors of LC incidence and LC-related mortality in ever smokers and never smokers, especially emphasizing cumulative $\mathrm{PM}_{2.5}$ exposure and individualized dietary habits; (2) to further investigate the factors that affect the survival of LC patients with a smoking habit vs. never smokers. Our findings provide evidence for further preventive strategy guidance.

\section{Materials and Methods}

\subsection{Study Design and Data Source}

The data for this retrospective, longitudinal follow-up cohort study were obtained from a population-based database of health examinations, the MJ Health Database (MJHD), from 2000 to 2015 in Taiwan. MJ is the name of the health check-up clinics and also the name of the database. The studied subjects were persons attending health check-ups, and they were distributed throughout the main island of Taiwan. The details of the MJHD have previously been described in the literature [20]. For each health examination visit, the results of physical examinations (anthropometric measurement and biological test data) and the results of self-administered questionnaires on lifestyle behaviors of participants were recorded. All participants signed an informed consent form before physical examination. We also linked the MJHD to Taiwan Cancer Registry (TCR) data and cause of death (COD) data for further evaluation of LC-related incidence and mortality. All participants were followed up with, from the baseline (i.e., the first health examination) until the endpoint (event of interest), the date of death, or the end of 2015. The study was approved by the Institutional Review Board (IRB) of Biomedical Science Research, Academia Sinica (IRB number: AS-IRB-BM-17044). 


\subsection{Selection of Study Population}

The selection process of the study population is shown in Figure 1; a total of 471,669 participants, who received at least once health examination, as shown in the MJHD, between 1 January 2000 and 31 December 2015, were enrolled. We first excluded 5208 participants with missing encrypted personal identification numbers (PIDNs), because this indicates a lack of follow-up. After linking the MJHD to the TCR and COD data through PIDNs, a total of 466,461 participants were included in the initial datasets.

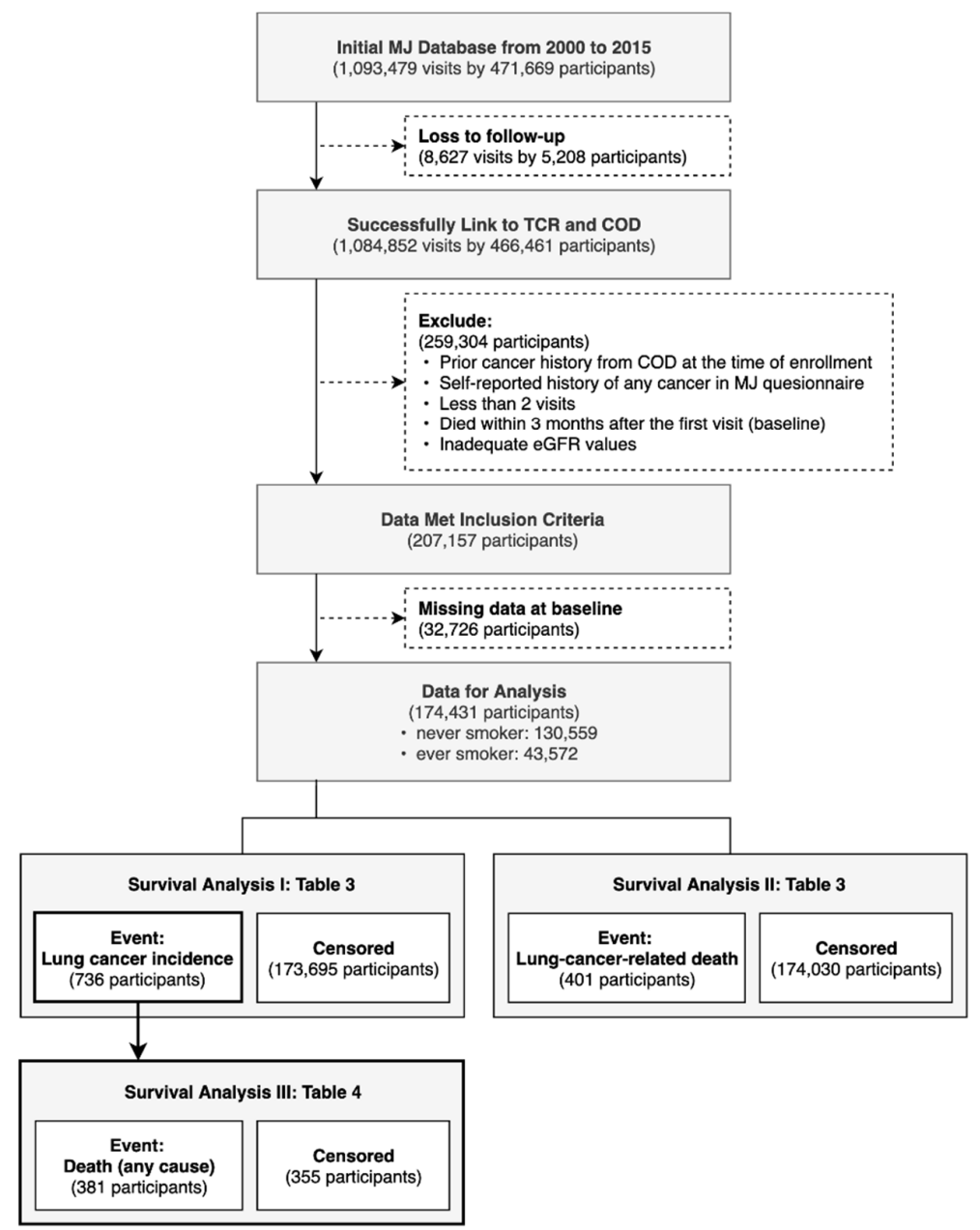

Figure 1. Flow Chart.

Participants with cancer diagnosis or self-reported history of any cancer prior to the date of health examination or who died within three months after the first visit were excluded. We also excluded participants who had missing values on baseline covariates or inadequate estimated glomerular filtration rate (eGFR) values (i.e., eGFR $<2$, eGFR $\geq 200$ ), or who provided fewer than two measuring points of eGFR during the study period, since at least two eGFR records were needed to compute an annual decline in eGFR. A total of 174,431 participants were enrolled for the final analysis, including 130,559 never smokers and 43,572 ever smokers.

\subsection{Endpoints}

For our first aim, the primary endpoint was an LC incident after the first health examination visit, which was identified from the TCR data using the International Classification of Diseases for Oncology, 3rd Edition (ICD-O-3) codes C33-C34. The secondary endpoint was LC-related death after the first health examination visit. We identified LC-related 
death from the COD data using the 9th revision (ICD-9) or the 10th revision (ICD-10) of the international classification of diseases (ICD-9 code 162; ICD-10 codes C33-C34).

As for our second aim, the endpoint was all-cause death of LC patients after diagnosis of LC, whose LC was diagnosed before 31 December 2014, and who were subsequently followed up until death or the end of 2015. The numbers of lung cancer cases diagnosed in different years during the studied period are listed in Table S1.

\subsection{Definition of Variables}

Information regarding participants' demographic characteristics, lifestyle habits, family history, comorbidities, and medical history were collected via standard self-administered questionnaires and health examination records.

Diabetic mellitus was defined as fasting glucose levels $>126 \mathrm{mg} / \mathrm{dL}$ or the current use of antihyperglycemic drugs. Hypertension, cardiovascular disease, and stroke were defined according to self-reported history or the current use of antihypertensive drugs and cardiac drugs. The estimated glomerular filtration rate (eGFR) was calculated from the modification of diet in renal disease (MDRD) equation [21]. The annual decline in eGFR was computed from the slope of the linear regression line of eGFR on the follow-up year. Both eGFR and CEA are common indicators in health examinations. In previous studies, eGFR decline might be associated with the incidence of some cancers, including urothelial cancer and lung cancer [22,23]. Carcinoembryonic antigen (CEA) was used as the surrogate biomarker of cancers, particularly for lung adenocarcinoma [24]. Never smokers were defined as those participants who self-reported never smoking. Participants who self-reported ever having smoked or smoking at least once a week were classified as ever smokers.

LC cases captured in our data were further classified into four types: small-cell carcinoma (ICD-O-3 morphology codes 8041, 8045), adenocarcinoma (8140, 8250, 8255, $8260,8550,8551$, and 8560), squamous cell carcinoma (8070, 8071, and 8072), and other carcinomas. LC stages were classified into four stages (I, II, III, and IV) according to the 6th and 7th editions of the American Joint Committee on Cancer (AJCC, https:/ / cancerstaging. org/references-tools/deskreferences/Pages/default.aspx, (accessed on 3 May 2021)).

$\mathrm{PM}_{2.5}$ exposure was estimated at each participant's address reported in the questionnaire coordinates by using a satellite-based spatiotemporal model [25] with a high spatial resolution of $1 \times 1 \mathrm{~km}$ on the basis of National Aeronautics and Space Administration (NASA) aerosol optical thickness (AOD) data [26]. The two-year mean $\mathrm{PM}_{2.5}$ concentration $\left(\mu \mathrm{g} / \mathrm{m}^{3}\right)$ prior to the heath examination date was used as an indicator of long-term exposure to ambient $\mathrm{PM}_{2.5}$ air pollution.

\subsection{Statistical Analysis}

Baseline characteristics of participants were presented as mean \pm standard deviation or median (interquartile range (IQR)) for continuous variables and frequency (percentage) for categorical variables. The intergroup difference of continuous variables was compared by using the independent t-test or Mann-Whitney $U$ test, depending on the normal distribution. A Chi-square test or Fisher's exact test was used to compare the intergroup difference of categorical variables, as appropriate.

To investigate the effect of $\mathrm{PM}_{2.5}$ and fruit or vegetable consumption on the incidence and mortality of lung cancer among all study participants, Cox proportional hazards regression models were used to estimate the hazard ratios (HR) and 95\% confidence intervals (95\% CI) after adjusting the related covariates (details in Supplementary File S1). The cut-off points for the servings of fruits and vegetables were obtained after statistical selection since statistical significance can be only seen at 2 servings after we applied different cut-off points from 2 to 5 . All statistical analyses were conducted with SAS software version 9.4 (SAS Institute, Cary, NC, USA). A $p$-value $\leq 0.05$ was considered statistically significant. 


\section{Results}

Overall, a total of $736(0.42 \%)$ LC cases were identified after a median follow-up of 12.3 years. The demographic data of the enrolled population are shown in Table 1. Compared to those who did not develop LC, LC patients were more likely to be older (55.0 vs. 39.0 years old, $p<0.001)$, and had a lower education level $(67.7 \%$ vs. $39.0 \%$, $p<0.001)$, poorer renal function (77.3 vs. $\left.86.5 \mathrm{~mL} / \mathrm{min} / 1.73 \mathrm{~m}^{2}, p<0.001\right)$, a higher level of carcinoembryonic antigen (CEA) ( 2.3 vs. $1.5 \mathrm{mg} / \mathrm{dL}, p<0.001$ ), greater family history of LC $(8.0 \%$ vs. $5.2 \%, p<0.001)$, and more comorbidities. Additionally, the LC group had more ever smokers ( $36.4 \%$ vs. $25.1 \%, p<0.001$ ) but lower $\mathrm{PM}_{2.5}$ exposure concentration (20.2 vs. $\left.21.5 \mu \mathrm{g} / \mathrm{m}^{3}, p<0.001\right)$ than those without LC.

Table 1. Baseline characteristics of enrolled population, stratified by the incidence of LC and LC-related mortality.

\begin{tabular}{|c|c|c|c|c|c|c|c|}
\hline \multirow{2}{*}{$\begin{array}{c}\text { Event of Interest } \\
\text { Variables }\end{array}$} & \multirow{2}{*}{$\begin{array}{c}\text { Overall } \\
(\mathrm{N}=\mathbf{1 7 4}, \mathbf{4 3 1})\end{array}$} & \multicolumn{3}{|c|}{ LC Incidence } & \multicolumn{3}{|c|}{ LC-Related Mortality } \\
\hline & & $\begin{array}{c}\text { Non LC } \\
(\mathrm{N}=173,695)\end{array}$ & $\begin{array}{c}\mathrm{LC} \\
(\mathrm{N}=736)\end{array}$ & $p$-Value & $\begin{array}{c}\text { Survivor } \\
(\mathrm{N}=174,030)\end{array}$ & $\begin{array}{l}\text { LC-Related Death } \\
\quad(\mathrm{N}=401)\end{array}$ & $p$-Value \\
\hline $\begin{array}{l}\text { Age (years), } \\
\text { mean } \pm S D\end{array}$ & $39.02 \pm 12.95$ & $38.95 \pm 12.92$ & $54.96 \pm 12.32$ & $<0.001$ & $38.97 \pm 12.92$ & $59.08 \pm 12.05$ & $<0.001$ \\
\hline Female, N (\%) & $87,722(50.29)$ & $87,363(50.30)$ & $359(48.78)$ & 0.411 & $87,563(50.31)$ & $159(39.65)$ & $<0.001$ \\
\hline \multicolumn{8}{|l|}{ Education level, N (\%) } \\
\hline High school or lower & $68,233(39.12)$ & $67,735(39.00)$ & $498(67.66)$ & $<0.001$ & $67,931(39.03)$ & $302(75.31)$ & $<0.001$ \\
\hline College or higher & $106,198(60.88)$ & $105,960(61.00)$ & $238(32.34)$ & & $106,099(60.97)$ & $99(24.69)$ & \\
\hline $\begin{array}{c}\text { Body mass index } \\
\left(\mathrm{kg} / \mathrm{m}^{2}\right), \text { mean } \pm \mathrm{SD}\end{array}$ & $22.91 \pm 3.60$ & $22.90 \pm 3.60$ & $23.39 \pm 3.31$ & $<0.001$ & $22.90 \pm 3.60$ & $23.53 \pm 3.40$ & $<0.001$ \\
\hline$<18.5$ & $16,147(9.26)$ & $16,111(9.28)$ & $36(4.89)$ & $<0.001$ & $16,128(9.27)$ & $19(4.74)$ & 0.005 \\
\hline $18.5-23.9$ & $97,271(55.76)$ & $96,853(55.76)$ & $418(56.79)$ & & $97,052(55.77)$ & $219(54.61)$ & \\
\hline $24-27.9$ & $46,721(26.78)$ & $46,501(26.77)$ & $220(29.89)$ & & $(26.77)$ & $128(31.92)$ & \\
\hline$\geq 28$ & $14,292(8.19)$ & $14,230(8.19)$ & $62(8.42)$ & & $14,257(8.19)$ & $35(8.73)$ & \\
\hline $\begin{array}{c}\text { eGFR } \\
\left(\mathrm{mL} / \mathrm{min} / 1.73 \mathrm{~m}^{2}\right) \\
\text { mean } \pm \mathrm{SD}, \mathrm{N}(\%)\end{array}$ & $86.41 \pm 17.83$ & $86.45 \pm 17.82$ & $77.31 \pm 15.91$ & $<0.001$ & $86.44 \pm 17.82$ & $74.44 \pm 15.50$ & $<0.001$ \\
\hline$\geq 90$ & $64,333(36.88)$ & $64,187(36.95)$ & $146(19.84)$ & $<0.001$ & $64,268(36.93)$ & $65(16.21)$ & $<0.001$ \\
\hline $60-89$ & $105,042(60.22)$ & $104,530(60.18)$ & $512(69.57)$ & & $104,771(60.20)$ & $271(67.58)$ & \\
\hline $45-59$ & $4471(2.56)$ & $4403(2.53)$ & $68(9.24)$ & & $4415(2.54)$ & $56(13.97)$ & \\
\hline$<45$ & $585(0.34)$ & $575(0.33)$ & $10(1.36)$ & & $576(0.33)$ & $9(2.24)$ & \\
\hline $\begin{array}{l}\text { eGFR annual decline } \\
\geq 5\end{array}$ & 20,385 (11.69) & $20,310(11.69)$ & 75 (10.19) & 0.205 & 20,346 (11.69) & $39(9.73)$ & 0.221 \\
\hline $\begin{array}{l}\mathrm{CEA}(\mathrm{mg} / \mathrm{dL}) \\
\text { mean } \pm \mathrm{SD}^{\mathrm{a}}\end{array}$ & $1.46 \pm 1.18$ & $1.45 \pm 1.45$ & $2.33 \pm 3.21$ & $<0.001$ & $1.45 \pm 1.17$ & $2.87 \pm 4.43$ & $<0.001$ \\
\hline $\begin{array}{l}\text { Family history of LC, } \\
\text { N (\%) }\end{array}$ & $9160(5.25)$ & $9101(5.24)$ & $59(8.02)$ & $<0.001$ & $9136(5.25)$ & $24(5.99)$ & 0.510 \\
\hline \multicolumn{8}{|l|}{ Comorbidities, N (\%) } \\
\hline Hypertension & $12,106(6.94)$ & $11,970(6.89)$ & $136(18.48)$ & $<0.001$ & $12,019(6.91)$ & $87(21.70)$ & $<0.001$ \\
\hline Diabetic mellitus & $7084(4.06)$ & $7020(4.04)$ & $64(8.70)$ & $<0.001$ & $7035(4.04)$ & 49 (12.22) & $<0.001$ \\
\hline CVD & $4754(2.73)$ & $4696(2.70)$ & $58(7.88)$ & $<0.001$ & $4710(2.71)$ & $44(10.97)$ & $<0.001$ \\
\hline CVA & $478(0.27)$ & $471(0.27)$ & $7(0.95)$ & 0.005 & $471(0.27)$ & $7(1.75)$ & $<0.001$ \\
\hline \multirow{2}{*}{\multicolumn{8}{|c|}{$\begin{array}{c}\text { Lifestyle behaviors, } \\
\text { N (\%) } \\
\text { Smoking status }\end{array}$}} \\
\hline & & & & & & & \\
\hline Never smoker & $130,559(74.85)$ & $130,091(74.90)$ & $468(63.59)$ & $<0.001$ & $130,344(74.90)$ & $215(53.62)$ & $<0.001$ \\
\hline Ever smoker & $43,872(25.15)$ & $43,604(25.10)$ & $268(36.41)$ & & $43,686(25.10)$ & $186(46.39)$ & \\
\hline $\begin{array}{l}\text { Fruit/vegetable } \geq 2 \\
\text { servings per day }\end{array}$ & 164,115 (94.09) & $163,430(94.09)$ & $685(93.07)$ & 0.242 & $163,744(94.09)$ & $371(92.52)$ & 0.183 \\
\hline $\begin{array}{c}\mathrm{PM}_{2.5} \text { exposure } \\
\left(\mu \mathrm{g} / \mathrm{m}^{3}\right), \\
\text { median } \pm \mathrm{IQR}, \mathrm{N}(\%)^{\mathrm{b}}\end{array}$ & $21.45 \pm 6.95$ & $21.45 \pm 6.95$ & $20.20 \pm 6.45$ & $<0.001$ & $21.45 \pm 6.95$ & $20.00 \pm 6.40$ & $<0.001$ \\
\hline$<17.95$ & $43,693(25.05)$ & $43,503(25.05)$ & $190(25.82)$ & & $43,572(25.04)$ & $121(30.17)$ & \\
\hline $17.95 \leq \mathrm{PM}_{2.5}<21.45$ & $43,671(25.04)$ & $43,413(24.99)$ & $258(35.05)$ & & $43,532(25.01)$ & $139(34.66)$ & \\
\hline $21.45 \leq \mathrm{PM}_{2.5}<24.9$ & $43,272(24.81)$ & $43,119(24.82)$ & $153(20.79)$ & & $43,194(24.82)$ & $78(19.45)$ & \\
\hline$\geq 24.9$ & $43,795(25.11)$ & $43,660(25.14)$ & 135 (18.34) & & $43,732(25.13)$ & $63(15.71)$ & \\
\hline $\begin{array}{l}\text { Follow-up time } \\
\quad \text { (years) }{ }^{c} \\
\text { median } \pm \text { IQR }\end{array}$ & $12.29 \pm 6.25^{c}$ & $12.29 \pm 6.24$ & $8.71 \pm 6.00$ & $<0.001$ & $12.29 \pm 6.25$ & $9.43 \pm 5.35$ & $<0.001$ \\
\hline $\begin{array}{l}\text { Type of LC diagnosis, } \\
\qquad N(\%)^{d}\end{array}$ & & & & & & & $<0.001$ \\
\hline Small-cell & $33(0.02)$ & & $33(4.48)$ & & $9(0.01)$ & $24(5.99)$ & \\
\hline
\end{tabular}


Table 1. Cont.

\begin{tabular}{|c|c|c|c|c|c|c|c|}
\hline \multirow{2}{*}{$\begin{array}{c}\text { Event of Interest } \\
\text { Variables }\end{array}$} & \multirow{2}{*}{$\begin{array}{c}\text { Overall } \\
(\mathrm{N}=174,431)\end{array}$} & \multicolumn{3}{|c|}{ LC Incidence } & \multicolumn{3}{|c|}{ LC-Related Mortality } \\
\hline & & $\begin{array}{c}\text { Non LC } \\
(N=173,695)\end{array}$ & $\begin{array}{c}\text { LC } \\
(\mathrm{N}=736)\end{array}$ & $p$-Value & $\begin{array}{c}\text { Survivor } \\
(\mathrm{N}=174,030)\end{array}$ & $\begin{array}{l}\text { LC-Related Death } \\
\quad(\mathrm{N}=401)\end{array}$ & $p$-Value \\
\hline Adenocarcinoma & $525(0.30)$ & & $525(71.33)$ & & $305(0.18)$ & $220(54.86)$ & \\
\hline Squamous cell & $76(0.04)$ & & 76 (10.33) & & $26(0.01)$ & $50(12.47)$ & \\
\hline Others & $102(0.06)$ & & $102(13.86)$ & & $39(0.02)$ & $63(15.71)$ & \\
\hline
\end{tabular}

Note: Values for categorical variables are presented as number (percentage), and continuous variables are presented as mean \pm standard deviation or median [1st interquartile-3rd interquartile]. $p$-Values are based on the Mann-Whitney $\mathrm{U}$ test for follow-up time and $\mathrm{PM}_{2.5}$ concentration; t-test for age, CEA level, BMI, and eGFR; or Pearson's chi-square test for eGFR annual decline, gender, education, BMI category, eGFR category, smoking status, intake of fruits and vegetables, proportions of family history of LC, type of LC diagnosis, and comorbidities. All statistical tests were two-sided. ${ }^{a}$ the normal range of CEA level is $<2.5 \mathrm{mg} / \mathrm{dL}$. ${ }^{\mathrm{b}} \mathrm{PM}_{2.5}$ is defined as the average of $\mathrm{PM}_{2.5}$ exposure in the two years prior to the enrollment date. The quartile cut-off points for $\mathrm{PM}_{2.5}$ were $\mathrm{PM}_{2.5}<17.95$ for $\mathrm{Q} 1 ; 17.95 \leq \mathrm{PM}_{2.5}<21.45$ for Q2; $21.45 \leq \mathrm{PM}_{2.5}<24.9$ for Q3; and $\mathrm{PM}_{2.5} \geq 24.9$ for Q4. ${ }^{c}$ the follow-up time was defined as the period from the baseline to the date of LC incidence, the end of follow-up (i.e., 2015/12/31), or death prior to 2015/12/31, whichever came first. ${ }^{d} 44$ people died of lung cancer-related causes without having an LC diagnosis record. LCs were classified into four types according to the ICD-O-3 morphology codes: (1) small-cell carcinoma (codes 8041, 8045); (2) adenocarcinoma (8140, 8250, 8255, 8260, 8550, 8551, and 8560); (3) squamous cell carcinoma (8070, 8071, and 8072) and (4) other carcinomas (remaining codes). Abbreviations: BMI, body mass index; CVD, cardiovascular disease CEA, carcinoembryonic antigen; CVA, cardiovascular accident; eGFR, estimated glomerular filtration rate; LC, lung cancer; PM, particulate matter.

The characteristics of LC patients are summarized in Table 2. Compared to the LC patients who never smoked, the ever smokers were likely to be older ( 57.9 vs. 53.3 years old, $p<0.001)$, and male $(90.3 \%$ vs. $28.9 \%, p<0.001)$, and had a lower education level (77.2\% vs. $62.2 \%, p<0.001$ ), higher CEA level (3.1 vs. $1.9 \mathrm{mg} / \mathrm{dL}, p<0.001)$, less fruit and vegetable intake $(87.3 \%$ vs. $96.4 \%, p<0.001)$ and less long-term $\mathrm{PM}_{2.5}$ exposure concentration ( $\left.20.0 \mathrm{vs} .20 .3 \mu \mathrm{g} / \mathrm{m}^{3}, p=0.012\right)$. Most of the LC patients without smoking habits were diagnosed with adenocarcinoma $(82.26 \%)$. The lag from health examination to LC diagnosis is not significantly different between the groups with different smoking habits $(p=0.631)$.

Table 2. Baseline characteristics of LC patients, stratified by smoking status.

\begin{tabular}{|c|c|c|c|c|}
\hline Variable & $\begin{array}{c}\text { Overall } \\
(n=736)\end{array}$ & $\begin{array}{l}\text { Never Smoker } \\
\quad(n=468)\end{array}$ & $\begin{array}{l}\text { Ever Smoker } \\
\quad(\mathrm{n}=268)\end{array}$ & $p$-Value \\
\hline Age (years), mean \pm SD & $54.96 \pm 12.32$ & $53.31 \pm 12.05$ & $57.85 \pm 12.28$ & $<0.001$ \\
\hline Female, $\mathrm{N}(\%)$ & $359(48.78)$ & $333(71.15)$ & $26(9.70)$ & $<0.001$ \\
\hline Education level, N(\%) & & & & $<0.001$ \\
\hline High school or lower & $498(67.66)$ & $291(62.18)$ & $207(77.24)$ & \\
\hline College or higher & $238(32.34)$ & $177(37.82)$ & $61(22.76)$ & \\
\hline Body mass index $\left(\mathrm{kg} / \mathrm{m}^{2}\right), \mathrm{N}(\%)$ & & & & 0.621 \\
\hline$<18.5$ & $36(4.89)$ & $23(4.91)$ & $13(4.85)$ & \\
\hline $18.5-23.9$ & $418(56.79)$ & $274(58.55)$ & $144(53.73)$ & \\
\hline $24-27.9$ & $220(29.89)$ & $133(28.42)$ & $87(32.46)$ & \\
\hline$\geq 28$ & $62(8.42)$ & $38(8.12)$ & $24(8.96)$ & \\
\hline $\mathrm{eGFR}\left(\mathrm{mL} / \mathrm{min} / 1.73 \mathrm{~m}^{2}\right), \mathrm{N}(\%)$ & & & & 0.021 \\
\hline$\geq 90$ & $146(19.84)$ & $103(22.01)$ & $43(16.04)$ & \\
\hline $60-89$ & $512(69.57)$ & $326(69.66)$ & $186(69.40)$ & \\
\hline $45-59$ & $68(9.24)$ & $35(7.48)$ & $33(12.31)$ & \\
\hline$<45$ & $10(1.36)$ & $4(0.85)$ & $6(2.24)$ & \\
\hline eGFR annual decline $\geq 5\left(\mathrm{~mL} / \mathrm{min} / 1.73 \mathrm{~m}^{2}\right), \mathrm{N}(\%)$ & $75(10.19)$ & $50(10.68)$ & $25(9.33)$ & 0.559 \\
\hline $\mathrm{CEA}^{\mathrm{a}}(\mathrm{mg} / \mathrm{dL})$, mean $\pm \mathrm{SD}$ & $2.33 \pm 3.21$ & $1.89 \pm 3.67$ & $3.10 \pm 1.97$ & $<0.001$ \\
\hline Family history of LC, N(\%) & $59(8.02)$ & $36(7.69)$ & $23(8.58)$ & 0.669 \\
\hline \multicolumn{5}{|l|}{ Comorbidities, $\mathrm{N}(\%)$} \\
\hline Hypertension & $136(18.48)$ & $83(17.74)$ & $53(19.78)$ & 0.492 \\
\hline Diabetic mellitus & $64(8.70)$ & $34(7.26)$ & $30(11.19)$ & 0.069 \\
\hline CVD & $58(7.88)$ & $38(8.12)$ & $20(7.46)$ & 0.750 \\
\hline CVA & $7(0.95)$ & $3(0.64)$ & $4(1.49)$ & 0.252 \\
\hline Fruit/vegetable $\geq 2$ servings per day, $\mathrm{N}(\%)$ & 685 (93.07) & 451 (96.37) & $234(87.31)$ & $<0.001$ \\
\hline $\mathrm{PM}_{2.5}$ exposure $\left(\mu \mathrm{g} / \mathrm{m}^{3}\right)$, median $\pm \mathrm{IQR}, \mathrm{N}(\%) \mathrm{b}$ & $20.20 \pm 6.45$ & $20.30 \pm 6.24$ & $20.00 \pm 7.77$ & 0.012 \\
\hline
\end{tabular}


Table 2. Cont.

\begin{tabular}{|c|c|c|c|c|}
\hline Variable & $\begin{array}{c}\text { Overall } \\
(n=736)\end{array}$ & $\begin{array}{l}\text { Never Smoker } \\
\quad(n=468)\end{array}$ & $\begin{array}{l}\text { Ever Smoker } \\
\quad(n=268)\end{array}$ & $p$-Value \\
\hline$<17.95$ & $190(25.82)$ & $106(22.65)$ & $84(31.34)$ & \\
\hline $17.95 \leq \mathrm{PM}_{2.5}<21.45$ & $258(35.05)$ & $164(35.04)$ & $94(35.07)$ & \\
\hline $21.45 \leq \mathrm{PM}_{2.5}<24.9$ & $153(20.79)$ & $106(22.65)$ & $47(17.54)$ & \\
\hline$\geq 24.9$ & $135(18.34)$ & $92(19.66)$ & $43(16.04)$ & \\
\hline $\begin{array}{l}\text { Time to event (years), median } \pm \text { IQR } \\
\text { Types of LC diagnosis, } N(\%)^{c}\end{array}$ & $8.71 \pm 6.00$ & $8.55 \pm 6.55$ & $8.93 \pm 5.36$ & $\begin{array}{c}0.631 \\
<0.001\end{array}$ \\
\hline Small-cell & $33(0.02)$ & $4(0.85)$ & $29(10.82)$ & \\
\hline Adenocarcinoma & $525(0.30)$ & $385(82.26)$ & $140(52.24)$ & \\
\hline Squamous cell & $76(0.04)$ & $20(4.27)$ & $56(20.90)$ & \\
\hline Others & $102(0.06)$ & $59(12.61)$ & $43(16.04)$ & \\
\hline All-cause death, $\mathrm{N}(\%)$ & $381(51.77)$ & $206(44.02)$ & $175(65.30)$ & $<0.001$ \\
\hline
\end{tabular}

Note: Values for categorical variables are presented as number (percentage), and continuous variables are presented as mean \pm standard deviation or median [1st interquartile-3rd interquartile]. $p$-values are based on the Mann-Whitney U test for time to event and PM $\mathrm{M}_{2.5}$ concentration; t-test for age and CEA level; or Pearson's chi-square test for eGFR annual decline, gender, education, BMI, eGFR category, intake of fruits and vegetables, proportions of family history of LC, type of LC diagnosis, and comorbidities. All statistical tests were two-sided. ${ }^{a}$ the normal range of CEA level is $<2.5 \mathrm{mg} / \mathrm{dL} .{ }^{b} \mathrm{PM}_{2.5}$ is defined as the average of $\mathrm{PM}_{2.5}$ exposure in the two years preceding the LC diagnosis date. The quartile cut-off points for $\mathrm{PM}_{2.5}<17.95$ for Q1; $17.95 \leq \mathrm{PM}_{2.5}<21.45$ for Q2; $21.45 \leq \mathrm{PM}_{2.5}<24.9$ for Q3; and $\mathrm{PM}_{2.5} \geq 24.9$ for $\mathrm{Q} 4{ }^{~}{ }^{\mathrm{C}} \mathrm{LCs}$ were classified into four types according to the ICD-O-3 morphology codes: (1) small-cell carcinoma (codes 8041 , 8045); (2) adenocarcinoma (8140, 8250, 8255, 8260, 8550, 8551, and 8560); (3) squamous cell carcinoma (8070, 8071, and 8072) and (4) other carcinomas (remaining codes). Abbreviations: BMI, body-mass index; CEA, carcinoembryonic antigen; CVA, cardiovascular accident; CVD, cardiovascular disease; eGFR, estimated glomerular filtration rate; LC, lung cancer; PM, particulate matter.

Table 3 shows the association between each factor and both LC incidence and LCrelated mortality. Ever smokers had higher cumulative incidences of LC development than never smokers ( $0.61 \%$ vs. $0.36 \%)$. When treating an LC incident as the event of interest, age greater than 50, lower education level, family history of LC, lower eGFR, and higher CEA level were found to be significant LC risk factors for both never smokers and ever smokers. For those who never smoked, being female was a significant risk factor for developing LC (aHR: 1.33, 95\%CI: 1.07-1.64, $p<0.01$ ), and a much higher proportion of adenocarcinoma was observed in never smokers than ever smokers ( $82.26 \%$ vs. $52.24 \%$, $p<0.001$, Table 2). This result is in-line with previous studies $[27,28]$. The effect of $\mathrm{PM}_{2.5}$ and vegetable and fruit intake on LC incidence differed between smokers and never smokers (Table 3 and Figure 2). For never smokers, the risk of developing LC rose significantly with every $10 \mu \mathrm{g} / \mathrm{m}^{3}$ increment of $\mathrm{PM}_{2.5}$ exposure (aHR: 1.32, 95\%CI: 1.12-1.56), while such an effect was not observed among ever smokers (aHR: 0.96, 95\%CI: 0.76-1.20). Conversely, consuming more than two servings of vegetables and fruits per day was able to help ever smokers reduce LC risk (aHR: $0.68,95 \% \mathrm{CI}$ : $0.47-0.97$ ), yet this was not seen in never smokers.

Table 3. Independent risk factors for LC incidence and LC-related mortality, stratified by smoking status.

\begin{tabular}{|c|c|c|c|c|c|c|c|c|}
\hline \multirow{3}{*}{$\begin{array}{c}\text { Event of Interest } \\
\text { Variables }\end{array}$} & \multicolumn{4}{|c|}{ LC Incidence } & \multicolumn{4}{|c|}{ LC-Related Mortality } \\
\hline & \multicolumn{2}{|c|}{$\begin{array}{c}\text { Never Smokers } \\
(\mathrm{N}=130,559 ; \mathrm{LC}=468)\end{array}$} & \multicolumn{2}{|c|}{$\begin{array}{c}\text { Ever Smokers } \\
(\mathrm{N}=43,872 ; \mathrm{LC}=268)\end{array}$} & \multicolumn{2}{|c|}{$\begin{array}{c}\text { Never Smokers } \\
(\mathrm{N}=130,559 ; \text { Death }=215)\end{array}$} & \multicolumn{2}{|c|}{$\begin{array}{c}\text { Ever Smokers } \\
(\mathrm{N}=43,872 ; \text { Death = 186) }\end{array}$} \\
\hline & aHR (95\%CI) & $p$-Value & aHR (95\%CI) & $p$-Value & aHR (95\%CI) & $p$-Value & aHR $(95 \% \mathrm{CI})$ & $p$-Value \\
\hline Age $\geq 50$ & $5.26(4.16,6.65)$ & $<0.001$ & $6.98(5.09,9.59)$ & $<0.001$ & $7.33(5.05,10.64)$ & $<0.001$ & $10.98(7.09,17.01)$ & $<0.001$ \\
\hline Female & $1.33(1.07,1.64)$ & 0.009 & $0.89(0.59,1.35)$ & 0.577 & $1.14(0.84,1.54)$ & 0.403 & $0.61(0.34,1.11)$ & 0.105 \\
\hline $\begin{array}{c}\text { Lower education } \\
\text { level } \\
\text { Body-mass index } \\
\left(\mathrm{kg} / \mathrm{m}^{2}\right)\end{array}$ & $1.05(0.84,1.31)$ & 0.653 & $1.86(1.37,2.54)$ & $<0.001$ & $1.15(0.82,1.61)$ & 0.422 & $2.33(1.54,3.53)$ & $<0.001$ \\
\hline $18.5-23.9$ & ref. & & ref. & & ref. & & ref. & \\
\hline$<18.5$ & $0.77(0.50,1.18)$ & 0.232 & $1.22(0.69,2.17)$ & 0.493 & $1.02(0.54,1.91)$ & 0.954 & $1.25(0.60,2.58)$ & 0.553 \\
\hline $24-27.9$ & $0.80(0.64,0.99)$ & 0.040 & $0.74(0.56,0.97)$ & 0.027 & $0.72(0.53,0.99)$ & 0.042 & $0.81(0.59,1.12)$ & 0.198 \\
\hline $\begin{array}{c}\geq 28 \\
\text { eGFR } \\
\left(\mathrm{mL} / \mathrm{min} / 1.73 \mathrm{~m}^{2}\right)\end{array}$ & $0.72(0.51,1.03)$ & 0.075 & $0.72(0.47,1.12)$ & 0.150 & $0.60(0.35,1.01)$ & 0.054 & $0.77(0.45,1.30)$ & 0.320 \\
\hline
\end{tabular}


Table 3. Cont.

\begin{tabular}{|c|c|c|c|c|c|c|c|c|}
\hline \multirow{3}{*}{$\begin{array}{c}\text { Event of Interest } \\
\text { Variables }\end{array}$} & \multicolumn{4}{|c|}{ LC Incidence } & \multicolumn{4}{|c|}{ LC-Related Mortality } \\
\hline & \multicolumn{2}{|c|}{$\begin{array}{c}\text { Never Smokers } \\
(\mathrm{N}=130,559 ; \mathrm{LC}=468)\end{array}$} & \multicolumn{2}{|c|}{$\begin{array}{c}\text { Ever Smokers } \\
(\mathrm{N}=43,872 ; \mathrm{LC}=268)\end{array}$} & \multicolumn{2}{|c|}{$\begin{array}{c}\text { Never Smokers } \\
(\mathrm{N}=130,559 ; \text { Death = 215) }\end{array}$} & \multicolumn{2}{|c|}{$\begin{array}{c}\text { Ever Smokers } \\
(\mathrm{N}=43,872 ; \text { Death = 186) }\end{array}$} \\
\hline & aHR $(95 \% C I)$ & $p$-Value & aHR (95\%CI) & $p$-Value & aHR $(95 \% \mathrm{CI})$ & $p$-Value & aHR $(95 \% \mathrm{CI})$ & $p$-Value \\
\hline$\geq 90$ & ref. & & ref. & & ref. & & ref. & \\
\hline $60-89$ & $1.58(1.25,2.00)$ & $<0.001$ & $1.33(0.93,1.89)$ & 0.117 & $1.88(1.28,2.77)$ & 0.001 & $1.10(0.71,1.68)$ & 0.676 \\
\hline $45-59$ & $1.85(1.23,2.79)$ & 0.003 & $1.87(1.15,3.06)$ & 0.012 & $3.13(1.81,5.40)$ & $<0.001$ & $1.92(1.11,3.33)$ & 0.020 \\
\hline$<45$ & $1.47(0.54,4.06)$ & 0.453 & $2.49(1.03,6.02)$ & 0.043 & $2.37(0.71,7.85)$ & 0.160 & $2.82(1.13,7.05)$ & 0.026 \\
\hline $\begin{array}{l}\text { eGFR annual decline } \\
\quad \geq 5\end{array}$ & $1.28(0.94,1.73)$ & 0.117 & $1.27(0.83,1.96)$ & 0.269 & $1.26(0.79,2.02)$ & 0.327 & $1.36(0.82,2.27)$ & 0.236 \\
\hline $\begin{array}{l}\mathrm{CEA}(\mathrm{mg} / \mathrm{dL}), \text { per } \\
\text { increment }\end{array}$ & $1.06(1.04,1.08)$ & $<0.001$ & $1.16(1.11,1.22)$ & $<0.001$ & $1.07(1.05,1.09)$ & $<0.001$ & $1.19(1.13,1.25)$ & $<0.001$ \\
\hline Family history of LC & $1.68(1.20,2.37)$ & $<0.001$ & $1.73(1.12,2.65)$ & 0.013 & $1.45(0.84,2.49)$ & 0.184 & $1.07(0.56,2.02)$ & 0.844 \\
\hline $\begin{array}{l}\text { Fruit/vegetable } \geq 2 \\
\text { servings per day }\end{array}$ & $1.12(0.69,1.82)$ & 0.648 & $0.68(0.47,0.97)$ & 0.034 & $0.92(0.47,1.80)$ & 0.813 & $0.76(0.48,1.20)$ & 0.241 \\
\hline $\begin{array}{c}\mathrm{PM}_{2.5} \text { exposure } \\
\left(\mu \mathrm{g} / \mathrm{m}^{3}\right)^{\mathrm{a}} \text {, per } 10 \\
\text { increment }\end{array}$ & $1.32(1.12,1.56)$ & 0.001 & $1.04(0.86,1.27)$ & 0.666 & $1.28(1.01,1.63)$ & 0.043 & $0.96(0.76,1.20)$ & 0.714 \\
\hline HTN & $1.17(0.90,1.52)$ & 0.256 & $1.25(0.90,1.74)$ & 0.190 & $1.17(0.81,1.68)$ & 0.402 & $1.10(0.74,1.61)$ & 0.638 \\
\hline DM & $0.92(0.64,1.33)$ & 0.663 & $0.99(0.67,1.47)$ & 0.948 & $1.35(0.87,2.09)$ & 0.176 & $0.99(0.63,1.54)$ & 0.954 \\
\hline CVA & $1.00(0.32,3.15)$ & 0.996 & $1.14(0.42,3.12)$ & 0.793 & $1.13(0.28,4.60)$ & 0.867 & $1.72(0.69,4.27)$ & 0.243 \\
\hline CVD & $1.37(0.96,1.94)$ & 0.079 & $1.14(0.71,1.83)$ & 0.594 & $1.70(1.09,2.64)$ & 0.019 & $1.42(0.86,2.34)$ & 0.166 \\
\hline
\end{tabular}

Note: The effect size of each variable was adjusted for the variables listed in Table 1 in each model. ${ }^{a} \mathrm{PM}_{2.5}$ is defined as the average of $\mathrm{PM}_{2.5}$ exposure in the two years prior to enrollment date. Abbreviations: aHR, adjusted hazard ratio; BMI, body-mass index; $\mathrm{CI}$, confidence interval; eGFR, estimated glomerular filtration rate; LC, LC; PM, particulate matter.

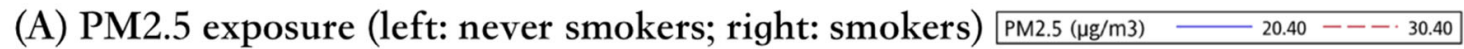
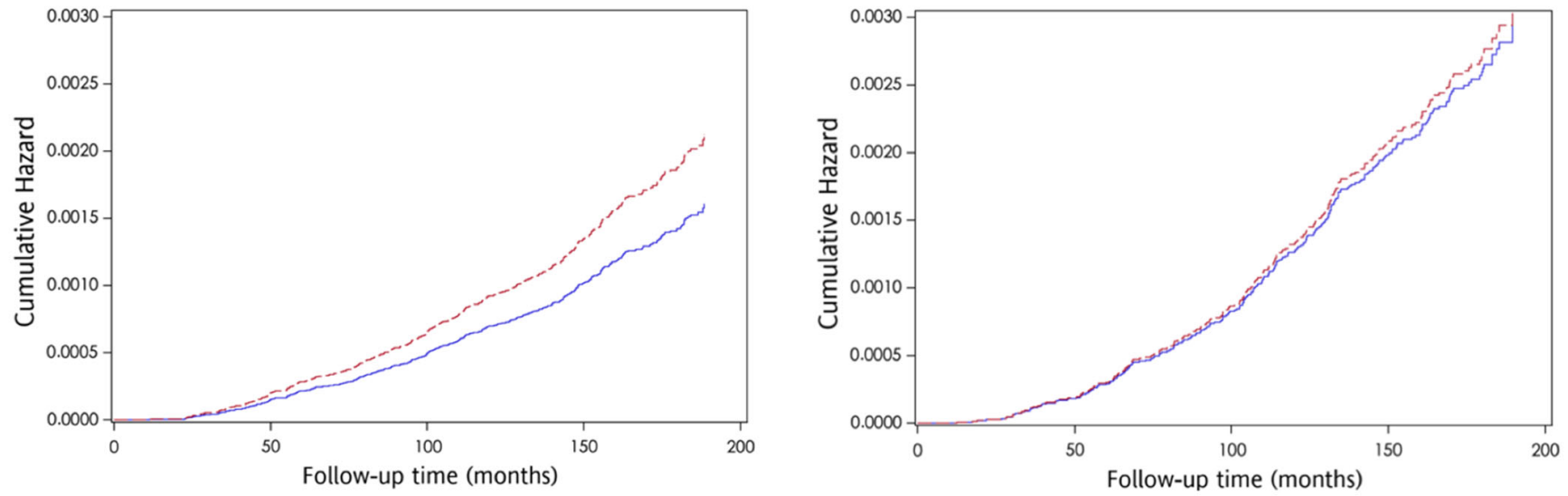

(B) Vegetable intake (left: never smokers; right: smokers) Fruit and vegetable intake $-<2$ senings per day $--->=2$ servings per day
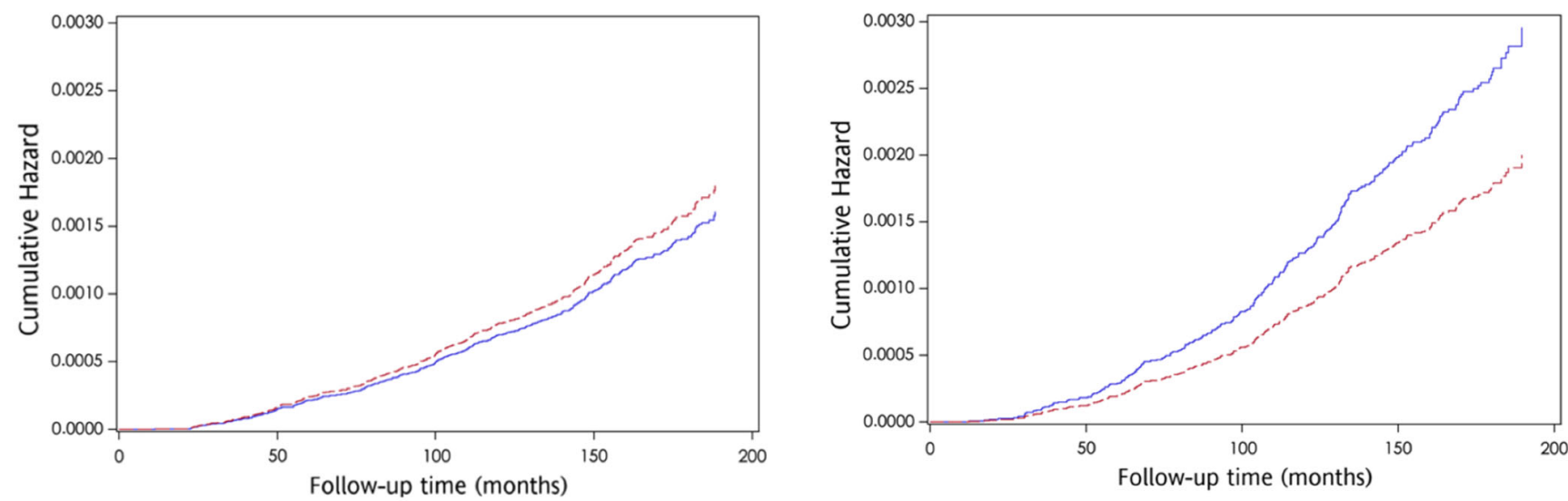

Figure 2. The effect of PM2.5 exposure (A) and vegetable intake (B) on cumulative hazard curve of LC incidence, stratified by smoking status. 
Regarding LC-related deaths in the whole population as the event of interest, a total of 401 death events $(0.23 \%)$ were reported after the median follow-up duration of 12.3 years (Table 1). Those who died of LC were older (59.1 vs. 39.0 years old, $p<0.001)$, had a lower educational level $(75.3 \%$ vs. $39.0 \%, p<0.001)$, were more ever smokers $(46.4 \%$ vs. $25.1 \%$, $p<0.001)$ and had more comorbidities at the baseline when compared to survivors; men accounted for around $60 \%$, and adenocarcinoma was the commonest type (Table 1 ). The LC-related mortality in ever smokers was 2.63 -fold higher than never smokers $(0.42 \%$ vs. $0.16 \%$ ), and the associations between risk factors and LC-related mortality are summarized in Table 3 and Figure 3. Every $10 \mu \mathrm{g} / \mathrm{m}^{3}$ increment of $\mathrm{PM}_{2.5}$ exposure concentration is a significant risk factor for LC-related mortality (aHR: 1.28, 95\% CI: 1.01-1.63, $p<0.05$ ) for never smokers, but not for ever smokers (aHR: 0.96, 95\%CI: 0.76-1.20).

\section{(A) PM2.5 exposure (left: never smokers; right: smokers)}
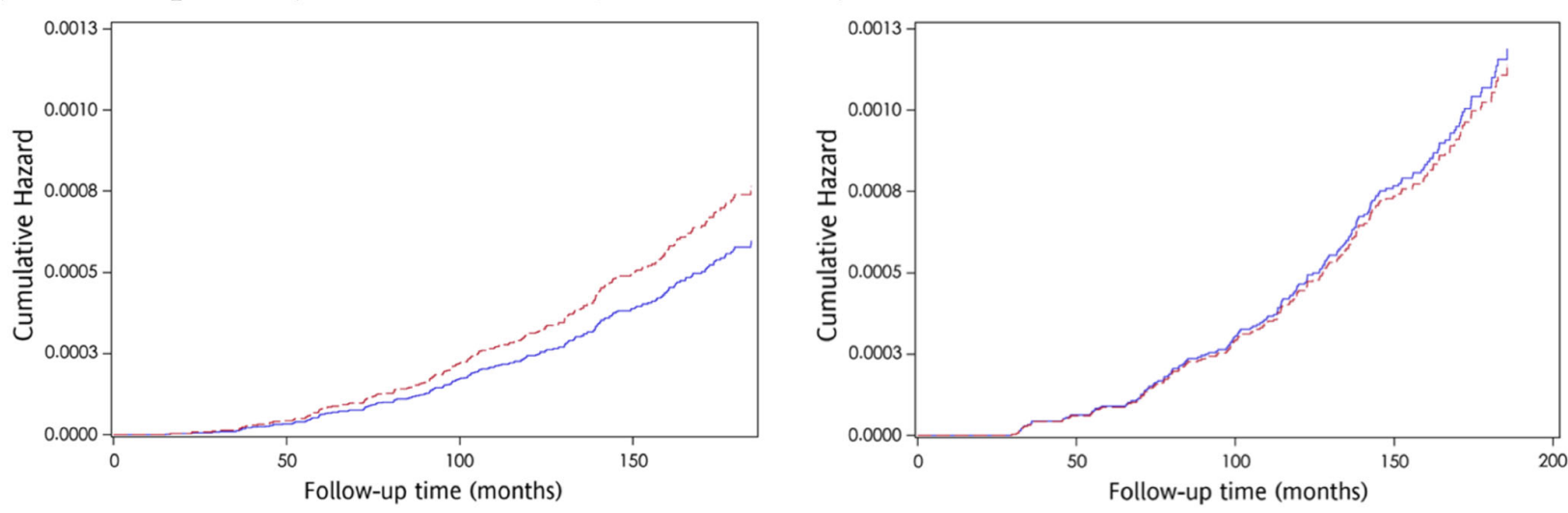

(B) Vegetable intake (left: never smokers; right: smokers) Fruit and vegetable intake $-<2$ senings per day $--->=2$ servings per day
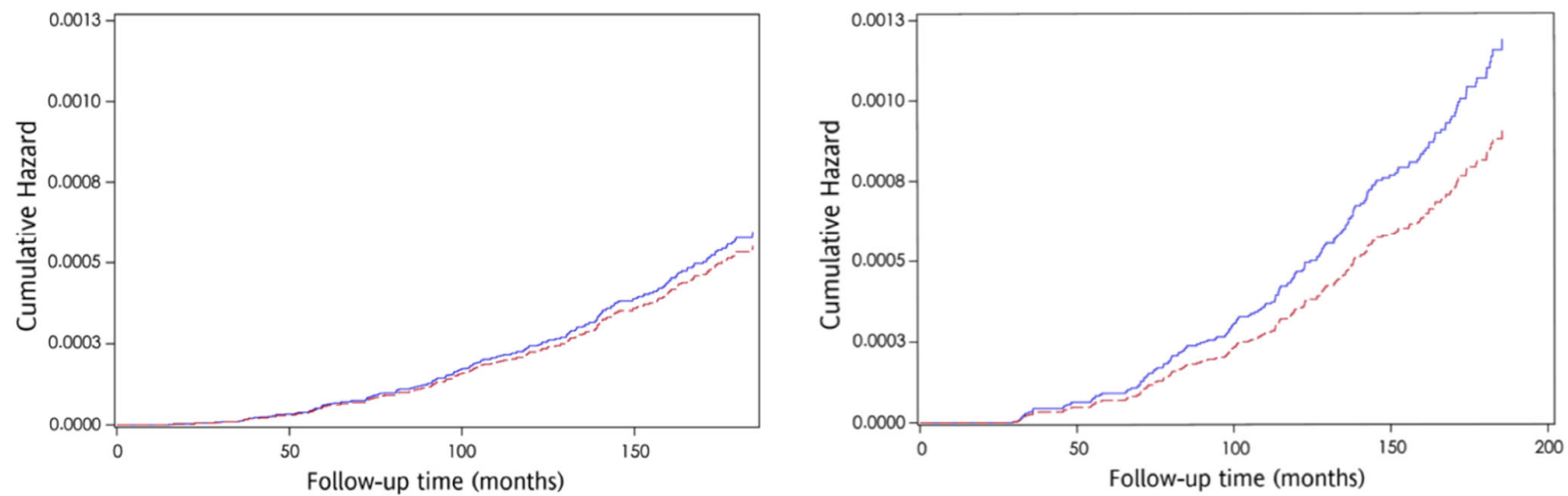

Figure 3. The effect of PM2.5 exposure (A) and vegetable intake (B) on cumulative hazard curve of LC-related death, stratified by smoking status.

Table 4 explores the association between risk factors and all-cause mortality for LC patients. Elderly age and advanced cancer stage at diagnosis of LC raised mortality risk in both never smokers (aHR: 1.03, 95\%CI: 1.01-1.04, $p<0.001$; aHR: 6.09, 95\%CI: 3.87-9.57, $p<0.001$ ) and ever smokers (aHR: 1.03, 95\%CI: 1.01-1.05, $p=0.001$; aHR: 7.48, 95\%CI: 4.15$13.48, p<0.001)$. For never smokers, non-adenocarcinoma type cancer (aHR: $2.55,95 \% \mathrm{CI}$ : $1.73-3.75, p<0.001)$ increased mortality. For the LC patients, regardless of their smoking status, $\mathrm{PM}_{2.5}$ no longer seems to be a significant risk factor for death. The relationship between $\mathrm{PM}_{2.5}$ and mortality is plotted in Figure 4. 
Table 4. Independent risk factors for all-cause mortality in LC patients, stratified by smoking status.

\begin{tabular}{|c|c|c|c|c|}
\hline \multirow[b]{2}{*}{ Variable } & \multicolumn{2}{|c|}{$\begin{array}{c}\text { Never Smoker }{ }^{a} \\
(\mathrm{~N}=323 ; \text { Death }=161)\end{array}$} & \multicolumn{2}{|c|}{$\begin{array}{c}\text { Ever Smoker } \\
(\mathrm{N}=192 ; \text { Death }=131)\end{array}$} \\
\hline & aHR (95\%CI) & $p$-Value & aHR (95\%CI) & $p$-Value \\
\hline Age at diagnosis of LC & $1.03(1.01,1.04)$ & $<0.001$ & $1.03(1.01,1.05)$ & 0.001 \\
\hline Female (ref. male) & $0.67(0.47,0.95)$ & 0.024 & $1.04(0.57,1.92)$ & 0.891 \\
\hline $\begin{array}{l}\text { Lower education level } \\
\text { (ref. college or higher) }\end{array}$ & $0.79(0.55,1.14)$ & 0.210 & $1.03(0.61,1.74)$ & 0.907 \\
\hline $\begin{array}{c}\text { Cancer stage }{ }^{\mathrm{b}} \text { III and IV } \\
\text { (ref. I and II) }\end{array}$ & $6.09(3.87,9.57)$ & $<0.001$ & $7.48(4.15,13.48)$ & $<0.001$ \\
\hline $\begin{array}{c}\text { Cancer type }{ }^{c} \\
\text { non-adeno(ref. } \\
\text { adenocarcinoma) }\end{array}$ & $2.55(1.73,3.75)$ & $<0.001$ & $1.29(0.90,1.86)$ & 0.165 \\
\hline $\begin{array}{l}\mathrm{PM}_{2.5} \text { exposure }{ }^{\mathrm{d}} \text {, per } \\
10 \text { increment, } \mu \mathrm{g} / \mathrm{m}^{3}\end{array}$ & $1.09(0.83,1.44)$ & 0.534 & $0.82(0.62,1.08)$ & 0.154 \\
\hline \multicolumn{5}{|c|}{ 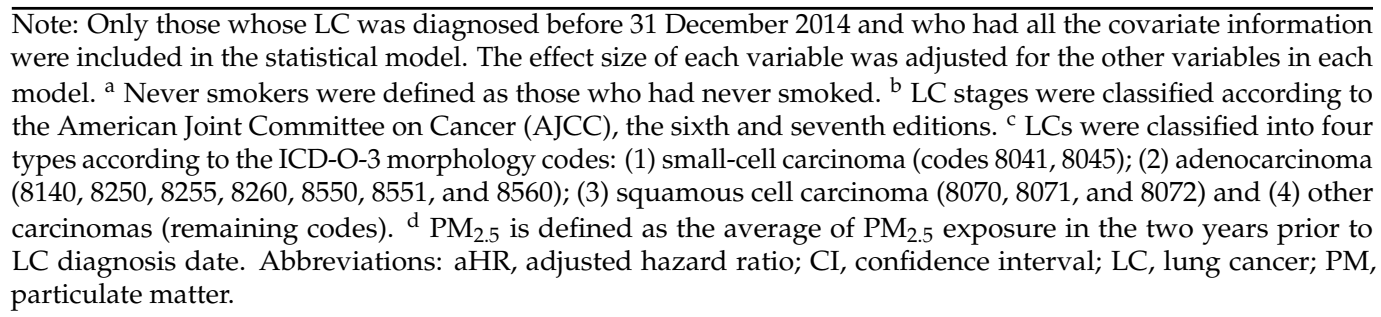 } \\
\hline
\end{tabular}

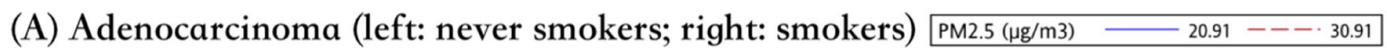
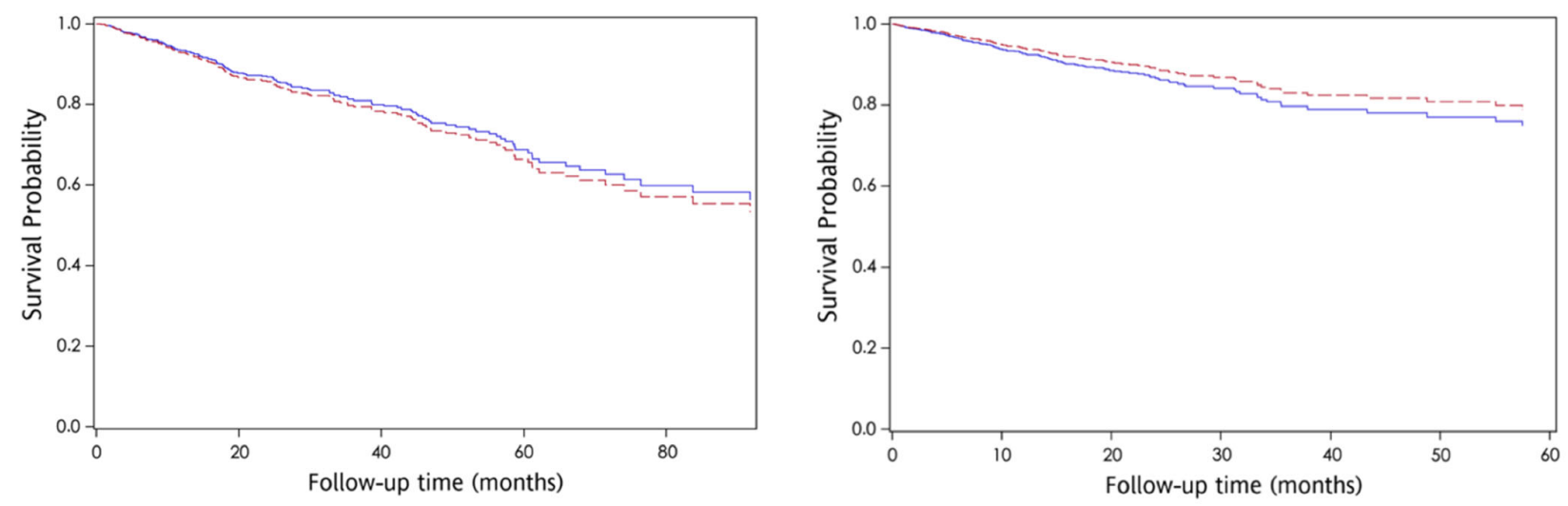

(B) Others (left: never smokers; right: smokers) $P$ PM2.5( $(\mathrm{kg} / \mathrm{m} 3)-20.91---\cdot 30.91$
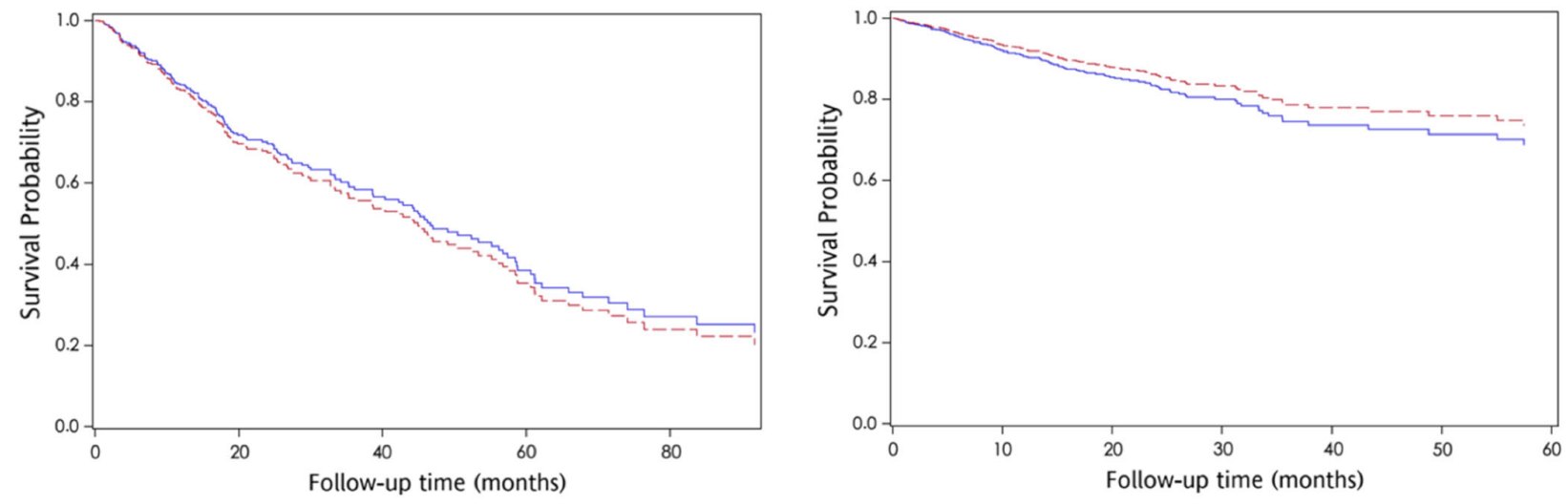

Figure 4. The effect of PM2.5 exposure on LC patients' survival, stratified by smoking status and cancer type ((A) adenocarcinoma and (B) others). 
A total of 736 participants developed LC during the studied period (Table S2). Among them, 72 participants did not have stage information of LC from the cancer registry. The numbers of LC cases from stage 1 to stage 4 were 201 (30.27\%), 30 (4.52\%), 121 (18.22\%), and $312(46.99 \%)$, respectively. Higher CEA levels were noted in ever smokers who also had more comorbidities, regardless of stages of lung cancer. A higher percentage of never smokers consumed $\geq 2$ servings of fruits and vegetables per day. The median follow-up durations from joining the database to lung cancer diagnosis were 9.5 years for the early stages of LC (stage $\leq 2$ ), and 8.31 years for later stages of LC (stage $\geq 3$ ), respectively.

\section{Discussion}

Several major findings were obtained from the current study. First, each 10-unit increment of cumulative $\mathrm{PM}_{2.5}$ exposure will increase the risk of LC incidence and LCrelated mortality by 1.32-fold and 1.28-fold in never smokers, respectively, but no such increase is seen in smokers. Second, daily consumption of at least two portions of fruits and vegetables decreased by $33 \%$ the risk of LC development in ever smokers, but not in never smokers.

The current study demonstrates a visible trend of cumulative $\mathrm{PM}_{2.5}$ exposure which increases by 1.32 the risk of LC incidence in never smokers, consistent with the results of previous meta-analyses showing a 1-46\% increase in the risk of LC incidence per $10 \mu \mathrm{g} / \mathrm{m}^{3}$ increase in $\mathrm{PM}_{2.5}$ concentration $[15,29,30]$. The interpretation of previous study results should be performed with caution, noting their geographical diversity and heterogeneous definitions of smoking status. Stronger associations between $\mathrm{PM}_{2.5}$ and LC in Asia than in North America and Europe have been reported [12,15]. The $\mathrm{PM}_{2.5}$ exposure concentration, with an average of $20.40 \mu \mathrm{g} / \mathrm{m}^{3}$, was higher in the current study than the levels reported in most previous Western studies which had average concentrations from 6.6 to $13.0 \mu \mathrm{g} / \mathrm{m}^{3}[13,29,30]$; this might be a potential contributor to higher incidences of LC in Taiwan. Several possible mechanisms for the related pathogenesis of $\mathrm{PM}_{2.5}$ and LC development have been proposed. Under $\mathrm{PM}_{2.5}$ long-term exposure, the epigenetic and microenvironmental alterations, mediated by microRNA dysregulation, DNA methylation, and cell autophagy and apoptosis, may activate oncogene-associated pathways to induce carcinomatosis of the lungs [31].

In contrast, the current study shows that the association between $\mathrm{PM}_{2.5}$ and $\mathrm{LC}$ risk was insignificant in ever smokers, perhaps because cigarette smoking leads to excess body weight, which may eliminate the effect of $\mathrm{PM}_{2.5}$. Regarding the risk of LC development, cigarette smoking increased the relative risk by a factor of 15 to 50 in current and ever smokers, respectively, whereas the relative risks reported for $\mathrm{PM}_{2.5}$ seldom exceeded 1.2 [32]. One study reported that LC risk increased by 32\% (95\% CI: 1.02, 1.69), $20 \%$ (95\% CI: $1.01,1.41)$ and $16 \%(95 \%$ CI: $1.02,1.30)$ per increase of $10 \mu \mathrm{g} / \mathrm{m}^{3} \mathrm{PM}_{2.5}$ in former, current, and never smokers, respectively [15].

Previous studies have reported that each increase of $10 \mu \mathrm{g} / \mathrm{m}^{3}$ in the ambient concentration of $\mathrm{PM}_{2.5}$ is associated with a 15 to $27 \%$ increase in mortality in LC patients, particularly for former and current smokers $[11,15]$. However, our study shows that the impact of $\mathrm{PM}_{2.5}$ increment on LC-related mortality can only be seen in never smokers, rather than in ever smokers. Effects from ambient $\mathrm{PM}_{2.5}$ on LC incidence and mortality among ever smokers were not observed because smoking produces polycyclic aromatic hydrocarbons ( $\mathrm{PAH})$ contained in $\mathrm{PM}_{2.5}$ and $\mathrm{PM}_{10}$ indoors, which are directly inhaled into the body and cause health impacts [33].

Furthermore, regardless of smoking status, elderly age and advanced cancer staging at diagnosis determined the poor outcomes after LC diagnosis rather than the accumulative $\mathrm{PM}_{2.5}$ exposure concentration. The lack of genetic mutation reporting of LC and detailed treatment might be a potential confounder for prognosis in LC patients.

Daily consumption of at least five portions ( $\geq 400 \mathrm{~g}$ ) of fruit and vegetables is recommended to reduce the risk of cardiovascular disease, cancer, and all-cause mortality [34,35]. The antioxidant activity contributed from biologically active compounds such as flavonoids, 
carotenoids, and other vitamins might be responsible for such risk reduction. However, in the literature there has not been adequate comprehensive evaluation of the protective role of fruits and vegetables in LC development and outcomes, and findings on whether they have a beneficial effect are mixed. A daily supplementation of $20-30 \mathrm{mg}$ of beta-carotene has been found to increase the incidence of LC among smokers and asbestos workers [36]. A meta-analysis showed an overall of $8 \%-18 \%$ risk reduction for LC with daily $70-300 \mathrm{~g}$ fruit and vegetable intake, while the protective effect was attenuated after stratifying by smoking status, with only a marginally significant association among current smokers and an insignificant inverse trend in former or never smokers [37]. In our study, we also found a protective effect against LC in ever smokers who consumed at least two portions of fruits and vegetables daily.

There are several limitations in this study. First, the current study lacks a standardized follow-up protocol for health condition monitoring, and there are potential confounders from self-reported questionnaire data. Though environmental tobacco smoking (ETS) is an important exposure risk for LC in non-smokers, we cannot quantify the ETS effect among non-smokers due to inadequate information from questionnaires, particularly for tobacco consumption amount and duration (packs per year). Second, the exact time point of LC diagnosis is uncertain because the pre-diagnostic information of LC patients could not be obtained. Third, the two-year $\mathrm{PM}_{2.5}$ concentration might not reflect a direct impact on the development of lung cancer. In our case, underestimation of the risk might have occurred because $\mathrm{PM}_{2.5}$ was declining during the studied period [38]. For participants who later joined the health check-up program, their exposure status might be lower than earlier participants. Fourth, these datasets ignored the effects of genetic mutation of LC and also ignored the potential effects of indoor air pollution and exposure to other carcinogens on outcomes of LC. Fifth, although we have considered geographical differences in our exposure model by addressing the participants' locations, the temporal resolution of exposure is in years. Therefore, it was hard to correlate with the community level characteristics and the variations of ambient exposure using this dataset. In southern Taiwan, the causes of serious air pollution can be attributed to three major factors including the hubs of the petrochemical industry, traffic-related air pollution, and downwind areas affecting the diffusion of air pollutants.

\section{Conclusions}

In conclusion, the strategy to lower LC incidence could differ by smoking status. For non-smokers, preventing long-term exposure to $\mathrm{PM}_{2.5}$ may attenuate the risk of LC development. Smoking cessation and encouraging daily consumption of at least two portions of fruits and vegetables is suggested for ever smokers.

Supplementary Materials: The following are available online at https:/ /www.mdpi.com/article/10 $.3390 /$ ijerph182312400/s1, Table S1: The number of lung cancer cases diagnosed by year from 2000 to 2015; Table S2: The descriptive statistics for the different stages of lung cancer cases; File S1: The detailed statistical analysis and variables' definitions.

Author Contributions: T.-C.C. and H.-L.H. conceived and designed the study. T.-C.C. and C.L. acquired the data. H.-L.H. and Y.-H.C. (Yung-Hsin Chuang) searched the literature. Y.-H.C. (YungHsin Chuang) and T.-H.L. analyzed the data. H.-L.H., T.-C.C., Y.-H.C. (Yen-Hsu Chen) and J.-Y.H. interpreted the results. H.-L.H., Y.-H.C. (Yung-Hsin Chuang) and T.-C.C. drafted the manuscript. All authors critically revised the manuscript. T.-C.C. obtained the funding and supervised this study. All authors have read and agreed to the published version of the manuscript.

Funding: This research was supported by a grant from the Ministry of Science and Technology, Taiwan (MOST- 108-2628-M-001-008-MY3).

Institutional Review Board Statement: Informed consent was obtained to authorize data processing and analysis. Ethical reviews were approved by the Institutional Review Board (IRB) of Biomedical Science Research, Academia Sinica (AS-IRB-BM-17044). Individually identifying data were removed and remained anonymous during the entire study. 
Informed Consent Statement: Informed consent was obtained from all subjects involved in the study.

Data Availability Statement: The data that support the findings of this study are available from the MJ Health Research Foundation and Ministry of Health and Welfare, Taiwan, but restrictions apply to the availability of these data, which were under approval for the current study and so are not publicly available. The linked data set used in this study had to be analyzed in person in the Health and Welfare Data Science Center, Ministry of Health and Welfare, Taiwan.

Acknowledgments: We would like to express our sincere gratitude to Kent M. Suárez for his English editing.

Conflicts of Interest: The authors declare no conflict of interest. The funders had no role in the design of the study, in the collection, analyses, or interpretation of data, in the writing of the manuscript, or in the decision to publish the results.

\section{References}

1. Siegel, R.L.; Miller, K.D.; Jemal, A. Cancer statistics, 2019. CA A Cancer J. Clin. 2019, 69, 7-34. [CrossRef] [PubMed]

2. Ministry of Health and Welfare. Cause of Death Statistics. Available online: https://www.mohw.gov.tw/lp-4650-2.html (accessed on 3 May 2021).

3. Giovino, G.A. Epidemiology of tobacco use in the United States. Oncogene 2002, 21, 7326-7340. [CrossRef]

4. Siegel, R.L.; Miller, K.D.; Jemal, A. Cancer statistics, 2020. CA Cancer J. Clin. 2020, 70, 7-30. [CrossRef] [PubMed]

5. Ferlay, J.; Soerjomataram, I.; Dikshit, R.; Eser, S.; Mathers, C.; Rebelo, M.; Parkin, D.M.; Forman, D.; Bray, F. Cancer Incidence and Mortality Worldwide: Sources, methods and major patterns in GLOBOCAN 2012. Int. J. Cancer 2015, 136, E359-E386. [CrossRef]

6. Couraud, S.; Debieuvre, D.; Moreau, L.; Dumont, P.; Margery, J.; Quoix, E.; Duvert, B.; Cellerin, L.; Baize, N.; Taviot, B.; et al. No impact of passive smoke on the somatic profile of lung cancers in never-smokers. Eur. Respir. J. 2015, 45, 1415-1425. [CrossRef]

7. Toh, C.-K.; Gao, F.; Lim, W.-T.; Leong, S.-S.; Fong, K.-W.; Yap, S.-P.; Hsu, A.A.; Eng, P.; Koong, H.-N.; Thirugnanam, A.; et al. Never-Smokers with Lung Cancer: Epidemiologic Evidence of a Distinct Disease Entity. J. Clin. Oncol. 2006, 24, $2245-2251$. [CrossRef] [PubMed]

8. Pallis, A.G.; Syrigos, K.N. Lung cancer in never smokers: Disease characteristics and risk factors. Crit. Rev. Oncol. Hematol. 2013, 88, 494-503. [CrossRef]

9. Bell, D.W.; Brannigan, B.W.; Matsuo, K.; Finkelstein, D.M.; Sordella, R.; Settleman, J.; Mitsudomi, T.; Haber, D.A. Increased Prevalence of EGFR-Mutant Lung Cancer in Women and in East Asian Populations: Analysis of Estrogen-Related Polymorphisms. Clin. Cancer Res. 2008, 14, 4079-4084. [CrossRef] [PubMed]

10. Tseng, C.-H.; Tsuang, B.-J.; Chiang, C.-J.; Ku, K.-C.; Tseng, J.-S.; Yang, T.-Y.; Hsu, K.-H.; Chen, K.-C.; Yu, S.-L.; Lee, W.-C.; et al. The Relationship between Air Pollution and Lung Cancer in Nonsmokers in Taiwan. J. Thorac. Oncol. 2019, 14, 784-792. [CrossRef]

11. Turner, M.C.; Krewski, D.; Pope, C.A., III; Chen, Y.; Gapstur, S.M.; Thun, M.J. Long-term ambient fine particulate matter air pollution and lung cancer in a large cohort of never-smokers. Am. J. Respir. Crit. Care Med. 2011, 184, 1374-1381. [CrossRef]

12. Hamra, G.B.; Guha, N.; Cohen, A.; Laden, F.; Raaschou-Nielsen, O.; Samet, J.M.; Vineis, P.; Forastiere, F.; Saldiva, P.; Yorifuji, T. Outdoor particulate matter exposure and lung cancer: A systematic review and meta-analysis. Environ. Health Perspect. 2014, 122, 9. [CrossRef] [PubMed]

13. Olsson, A.C.; Gustavsson, P.; Kromhout, H.; Peters, S.; Vermeulen, R.; Brüske, I.; Pesch, B.; Siemiatycki, J.; Pintos, J.; Brüning, T.; et al. Exposure to Diesel Motor Exhaust and Lung Cancer Risk in a Pooled Analysis from Case-Control Studies in Europe and Canada. Am. J. Respir. Crit. Care Med. 2011, 183, 941-948. [CrossRef]

14. Yorifuji, T.; Kashima, S.; Tsuda, T.; Ishikawa-Takata, K.; Ohta, T.; Tsuruta, K.-I.; Doi, H. Long-term exposure to traffic-related air pollution and the risk of death from hemorrhagic stroke and lung cancer in Shizuoka, Japan. Sci. Total Environ. 2013, 443, 397-402. [CrossRef]

15. Huang, F.; Pan, B.; Wu, J.; Chen, E.; Chen, L. Relationship between exposure to PM2.5 and lung cancer incidence and mortality: A meta-analysis. Oncotarget 2017, 8, 43322-43331. [CrossRef]

16. Ito, Y.; Wakai, K.; Suzuki, K.; Ozasa, K.; Watanabe, Y.; Seki, N.; Ando, M.; Nishino, Y.; Kondo, T.; Ohno, Y.; et al. Lung Cancer Mortality and Serum Levels of Carotenoids, Retinol, Tocopherols, and Folic Acid in Men and Women: A Case-Control Study Nested in the JACC Study. J. Epidemiol. 2005, 15, S140-S149. [CrossRef] [PubMed]

17. Lei, Y.; Cai, W.; Chen, Y.; Du, Y.-X. Some lifestyle factors in human lung cancer: A case-control study of 792 lung cancer cases. Lung Cancer 1996, 14, S121-S136. [CrossRef]

18. Gao, Y. Risk factors for lung cancer among nonsmokers with emphasis on lifestyle factors. Lung Cancer 1996, 14, S39-S45. [CrossRef]

19. Blumberg, J. The Alpha-Tocopherol, Beta-Carotene Cancer Prevention Study in Finland. Nutr. Rev. 2009, 52, 242-245. [CrossRef] [PubMed]

20. Wu, X.; Tsai, S.P.; Tsao, C.K.; Chiu, M.L.; Tsai, M.K.; Lu, P.J.; Lee, J.H.; Chen, C.H.; Wen, C.; Chang, S.-S.; et al. Cohort Profile: The Taiwan MJ Cohort: Half a million Chinese with repeated health surveillance data. Int. J. Epidemiol. 2017, 46, 1744-1744g. [CrossRef] 
21. Levey, A.S.; Bosch, J.P.; Lewis, J.B.; Greene, T.; Rogers, N.; Roth, D.R.; the Modification of Diet in Renal Disease Study Group. A More Accurate Method to Estimate Glomerular Filtration Rate from Serum Creatinine: A New Prediction Equation. Ann. Intern. Med. 1999, 130, 461-470. [CrossRef]

22. Mok, Y.; Ballew, S.H.; Sang, Y.; Coresh, J.; Joshu, C.E.; Platz, E.A.; Matsushita, K. Albuminuria, Kidney Function, and Cancer Risk in the Community. Am. J. Epidemiol. 2020, 189, 942-950. [CrossRef] [PubMed]

23. Chuang, Y.-H.; Lin, I.-F.; Lao, X.Q.; Lin, C.; Chan, T.-C. The Association between Renal Function Decline and the Incidence of Urothelial Carcinoma: A 16-year Retrospective Cohort Study in Taiwan. Eur. Urol. Open Sci. 2021, 27, 1-9. [CrossRef] [PubMed]

24. Grunnet, M.; Sorensen, J. Carcinoembryonic antigen (CEA) as tumor marker in lung cancer. Lung Cancer 2012, 76, 138-143. [CrossRef] [PubMed]

25. Lin, C.; Li, Y.; Yuan, Z.; Lau, A.; Li, C.; Fung, J. Using satellite remote sensing data to estimate the high-resolution distribution of ground-level PM2.5. Remote Sens. Environ. 2015, 156, 117-128. [CrossRef]

26. Zhang, Z.; Chang, L.-Y.; Lau, A.K.H.; Chan, T.-C.; Chuang, Y.C.; Chan, J.; Lin, C.; Jiang, W.K.; Dear, K.; Zee, B.C.-Y.; et al. Satellitebased estimates of long-term exposure to fine particulate matter are associated with C-reactive protein in 30,034 Taiwanese adults. Int. J. Epidemiol. 2017, 46, 1126-1136. [CrossRef] [PubMed]

27. Yun, Y.D.; Back, J.H.; Ghang, H.; Jee, S.H.; Kim, Y.; Lee, S.M.; Samet, J.M.; Lee, K.S. Hazard Ratio of Smoking on Lung Cancer in Korea According to Histological Type and Gender. Lung 2015, 194, 281-289. [CrossRef]

28. Khuder, S.A. Effect of cigarette smoking on major histological types of lung cancer: A meta-analysis. Lung Cancer 2001, 31, 139-148. [CrossRef]

29. Ghazipura, M.; Garshick, E.; Cromar, K. Ambient PM 2.5 exposure and risk of lung cancer incidence in North America and Europe. Environ. Res. Commun. 2019, 1, 015004. [CrossRef]

30. Raaschou-Nielsen, O.; Andersen, Z.J.; Beelen, R.; Samoli, E.; Stafoggia, M.; Weinmayr, G.; Hoffmann, B.; Fischer, P.; Nieuwenhuijsen, M.J.; Brunekreef, B.; et al. Air pollution and lung cancer incidence in 17 European cohorts: Prospective analyses from the European Study of Cohorts for Air Pollution Effects (ESCAPE). Lancet Oncol. 2013, 14, 813-822. [CrossRef]

31. Li, R.; Zhou, R.; Zhang, J. Function of PM2.5 in the pathogenesis of lung cancer and chronic airway inflammatory diseases (Review). Oncol. Lett. 2018, 15, 7506-7514. [CrossRef]

32. Lipfert, F.W.; Wyzga, R.E. Longitudinal relationships between lung cancer mortality rates, smoking, and ambient air quality: A comprehensive review and analysis. Crit. Rev. Toxicol. 2019, 49, 790-818. [CrossRef] [PubMed]

33. Slezakova, K.; Castro, D.; Pereira, M.; Morais, S.; Delerue-Matos, C.; Alvim-Ferraz, M. Influence of tobacco smoke on carcinogenic PAH composition in indoor PM10 and PM2.5. Atmos. Environ. 2009, 43, 6376-6382. [CrossRef]

34. WHO. Global Strategy on Diet, Physical Activity and Health. Available online: http://www.who.int/dietphysicalactivity/fruit/ en / (accessed on 10 May 2021).

35. Aune, D.; Giovannucci, E.; Boffetta, P.; Fadnes, L.T.; Keum, N.N.; Norat, T.; Greenwood, D.C.; Riboli, E.; Vatten, L.J.; Tonstad, S. Fruit and vegetable intake and the risk of cardiovascular disease, total cancer and all-cause mortality-A systematic review and dose-response meta-analysis of prospective studies. Int. J. Epidemiol. 2017, 46, 1029-1056. [CrossRef] [PubMed]

36. Druesne-Pecollo, N.; Latino-Martel, P.; Norat, T.; Barrandon, E.; Bertrais, S.; Galan, P.; Hercberg, S. Beta-carotene supplementation and cancer risk: A systematic review and metaanalysis of randomized controlled trials. Int. J. Cancer 2009, 127, 172-184. [CrossRef] [PubMed]

37. Vieira, A.R.; Abar, L.; Vingeliene, S.; Chan, D.S.M.; Aune, D.; Navarro-Rosenblatt, D.; Stevens, C.; Greenwood, D.; Norat, T. Fruits, vegetables and lung cancer risk: A systematic review and meta-analysis. Ann. Oncol. 2016, 27, 81-96. [CrossRef] [PubMed]

38. Cheng, F.-Y.; Hsu, C.-H. Long-term variations in PM2.5 concentrations under changing meteorological conditions in Taiwan. Sci. Rep. 2019, 9, 6635. [CrossRef] [PubMed] 